\title{
Fabrication of membranes and microchannels in low temperature co-fired ceramic (LTCC) substrate using novel water-based sacrificial carbon pastes
}

\author{
Karol Malecha \\ Wrocław University of Technology, Faculty of Microsystem Electronics and Photonics, \\ Wybrzeże S. Wyspiańskiego 27, 50-370 Wrocław, Poland \\ karol.malecha@pwr.wroc.pl \\ Thomas Maeder and Caroline Jacq \\ École Polytechnique Fédérale de Lausanne (EPFL), Laboratoire de Production \\ Microtechnique (LPM), CH-1015 Lausanne, Switzerland \\ thomas.maeder@epfl.ch caroline.jacq@epfl.ch
}

Published version: Malecha-K Maeder-T Jacq-C, "Fabrication of membranes and microchannels in low-temperature co-fired ceramic (LTCC) substrate using novel water-based sacrificial carbon pastes", Journal of the European Ceramic Society 32 (12), 3277-3286, 2012.

Permanent link: http://hdl.handle.net/10.1016/j.jeurceramsoc.2012.04.036

\begin{abstract}
In this work, 3D structuration of LTCC (Low-Temperature Co-fired Ceramic) for microfluidics was studied, using two novel sacrificial carbon paste compositions. These pastes are based on graphite with a water-soluble vehicle consisting of polyvinylpyrrolidone binder (PVP) dissolved in propylene glycol (PG), which is not aggressive to green LTCC material. Both examined pastes differ slightly in binder content and added plasticizer, glycerol $(\mathrm{G})$ or trimethylolpropane (TMP). The thermal properties of the sacrificial carbon pastes have been examined using combined thermo-gravimetric analysis (TGA), differential thermal analysis (DTA) and differential thermo-gravimetry (DTG). The sacrificial carbon pastes have been applied to the fabrication of membranes and microchannels in LTCC substrate. A comparison of the obtained features has been made using X-ray tomography and optical profile measurements. Moreover, changes in the composition of the fired LTCC material after cofiring have been studied using X-ray photoelectron spectroscopy (XPS) and surface wettability measurements.
\end{abstract}

Keywords: LTCC, membrane, microchannel, carbon sacrificial paste. 


\section{Introduction}

Low temperature co-fired ceramics (LTCC) is a well known technology, which has been used for fabrication of multilayer substrates and hybrid circuits. Those structures are called multichip ceramic modules (MCM-C) [1]. They are built of several dielectric tapes that are mechanically and electrically connected. Electrical interconnections are provided by networks of conductive lines ( $\mathrm{Ag}, \mathrm{Au}, \mathrm{Ag}-\mathrm{Pd}$ ), as well as surface and buried passives, which usually are deposited onto green or fired LTCC material using the standard screen-printing method. An intimate mechanical connection between LTCC tapes is formed during the lamination and cofiring processes. During lamination, the LTCC tapes are stacked together in a proper order and then compressed at high pressure (10-30 MPa) and elevated temperature $\left(40-90{ }^{\circ} \mathrm{C}\right)$. Typically, this lamination step lasts 1-20 min. Next, the LTCC module is cofired at a two-step thermal profile with a peak temperature of $850-900{ }^{\circ} \mathrm{C}$. Fired LTCC material usually has very good temperature stability (up to $650{ }^{\circ} \mathrm{C}$ ) $[2,3]$, and some compositions also exhibit a high degree of chemical resistance, making them suitable for fabrication of chemical microreactors, even for aggressive substances [4]. Finally, its CTE (coefficient of thermal expansion) is reasonably well matched to that of silicon [5]. Thanks to all these advantages, LTCC technology is well established in both low-volume, high-performance and highvolume, low-cost applications. In the last several years, LTCC technology has been applied to the fabrication of miniature sensors, actuators and microfluidic systems [6-8]. These LTCCbased microsystems consist of various micromechanical (e.g. membranes) or microfluidic (microchannels) structures. However, the quality of the final micromechanical or microfluidic structure is determined by the lamination and co-firing steps. The corresponding high applied pressures and temperatures during these steps may cause deformation of the fabricated spatial structures and precludes realization of structures with complex geometry inside LTCC multilayer modules. Several methods have been proposed to preserve the desired geometry of intricate micromechanical or microfluidic features [9-12], with the most common technique basing on various sacrificial volume materials (SVMs). Various types of substances can be used as an SVM, e.g. wax, graphite, polymers or mineral materials [13-17]. Commercially available thick-film pastes are usually formulated using terpineol or dibutyl carbitol as solvents for the organic vehicle [18], which are aggressive to the LTCC binder of most commercial compositions [19]. In order to eliminate this problem, we have proposed a graphite sacrificial paste with a polyvinyl alcohol (PVA) dissolved in a propylene glycol (PG)-glycerol (G)-water vehicle [19, 20]. However, we have noticed that the viscosity of the PVA-PG-G-water-based SVM paste tends to increase over time. This tendency to gelling of the PVA-PG-G-water binder was ascribed to loss of water, which kept polyvinyl alcohol in solution. In this work, in order to address this issue, we sought a new water-soluble binder that - in contrast to PVA - is intrinsically soluble in polar solvents such as PG, and selected polyvinylpyrrolidone (PVP). After preliminary studies, two graphite sacrificial pastes based on PVP dissolved in PG have been formulated and studied in detail. Both pastes differ in binder content and the added plasticizer, glycerol (G) or trimethylopropane (TMP). Both new SVM compositions do not exhibit gelling. Moreover, the applied PVP-PG-G and PVP-PG- 
TMP vehicles essentially eliminate the problem of dissolution of green LTCC material, which is especially crucial for very thin $(\sim 50 \mu \mathrm{m})$ tapes.

The properties of the PVP-PG-G, PVP-PG-TMP-based sacrificial pastes and green LTCC tape were investigated using thermo-gravimetric analysis (TGA), differential thermal analysis (DTA) and differential thermo-gravimetry (DTG). The new SVM compositions were applied to fabrication of $50 \mu \mathrm{m}$ thin membranes and microchannels. The quality of the resulting structures was studied using scanning electron microscopy (SEM), X-ray tomography and optical profile measurement gauge. Influence of the applied SVM paste on surface morphology and atomic composition of the LTCC material after co-firing were investigated using x-ray photoelectron spectroscopy (XPS) and contact angle measurement.

\section{Experimental}

\subsection{Paste preparation}

The solvents, PVP binders and plasticizers used in this study were all purchased from SigmaAldrich and used without further purification:

- Solvents: propylene glycol (PG, 1,2-propanediol) or butylene glycol (BG, 2,3butanediol)

- Binders: polyvinylpyrrolidone (PVP-40 or PVP-360, respectively 40 or $360 \mathrm{kDa}$ average molecular weight)

- Plasticizers: glycerol (G, 1,2,3-propanetriol), trimethylolpropane (TMP, 2-ethyl-1hydroxymethyl-1,3-propanediol) or sorbitol (one isomer of 1,2,3,4,5,6-hexanehexol)

The vehicles were prepared by dissolving the binder under agitation and moderate heat (ca. $80^{\circ} \mathrm{C}$ ) in the solvent-plasticizer mixture. Following this, the resulting vehicles and the graphite powder (TIMCAL TIMREX KS5-25, used throughout this study) were mixed using a three-roll mill (EXAKT 50), yielding a series of screen-printable inks.

\subsection{Preliminary evaluation of pastes}

In a first stage, different PVP-based vehicles were screened for printability and compatibility with LTCC tape, to select the most promising variants that were later used in the more detailed studies. To this end, the inks were printed onto DuPont (DP) 951 LTCC tapes, including thin $50 \mu \mathrm{m}$ tapes (DP 951C2), and qualitatively evaluated according to three criteria: (1) print quality, (2) aggressiveness to LTCC tape and (3) burnout compatibility with LTCC.

\subsection{Pastes used for detailed studies}

Both sacrificial pastes were based on the same binder, PVP-360, dissolved in PG with two different plasticizers, yielding the compositions $8 \% \mathrm{PVP}+88 \% \mathrm{PG}+4 \% \mathrm{G}$, and $9 \% \mathrm{PVP}+$ $89 \% \mathrm{PG}+2 \% \mathrm{TMP}$. The corresponding inks consisted of $60 \%$ vehicle $+40 \% \mathrm{KS} 5-25$ carbon 
powder for PVP-PG-G, and 66\% vehicle + 34\% KS5-25 for PVP-PG-TMP (all compositions given on a weight basis).

\subsection{Thermal analyses of the sacrificial pastes and applied LTCC material}

Thermal characterization of the PVP-PG-G, PVP-PG-TMP-based sacrificial pastes and DP951 LTCC tape was made using combined TGA/DTG/DTA analysis. All thermal analyses were performed in air atmosphere with a heating rate of $5^{\circ} \mathrm{C} / \mathrm{min}$. Results of the TGA/DTG/DTA analysis for all investigated materials are presented in Figs. 1-3. Weight changes of the samples with temperature are illustrated by red dotted line (TG curve). Rate of the sample weight loss in the specific temperature is presented by black solid line (DTG curve). The DTA curve - dashed blue line - shows measured heat flow. As can be seen in Fig. 1, organic vehicle of the DP951 LTCC burnouts in two stages yielding a total weight loss of about $12 \%$. Burnout of the LTCC organic vehicle proceeds in the temperature ranges from $175^{\circ} \mathrm{C}$ to $250^{\circ} \mathrm{C}$ and from $250^{\circ} \mathrm{C}$ to $350^{\circ} \mathrm{C}$ with two well separated peaks at the DTG curve. The exothermal nature of the LTCC organic binder decomposition process is confirmed by two maximums at the DTA curve. Weight of the DP951 LTCC tape remains constant for temperature above ca. $450^{\circ} \mathrm{C}$. Thermal behavior of the PVP-PG-G and PVP-PG-TMP-based sacrificial pastes is very similar. Decomposition of both sacrificial pastes takes place in three stages. In the first stage, from $100^{\circ} \mathrm{C}$ to $200^{\circ} \mathrm{C}$, the solvents and plasticizer evaporate rapidly, without well separated peaks at the DTA curves (minimum at $155^{\circ} \mathrm{C}$ ). This process is connected with main weight loss (60-65\%). Minimum at the DTA curves confirms endothermal nature of the organics evaporation process. The non-volatile components of the organic vehicle (mainly PVA binder) are decomposed in the second stage which occurs in temperature up to $350^{\circ} \mathrm{C}$.

Thermal decomposition of the PVA binder is linked with a small weight loss (2-3\%), and is illustrated by a small peak on the DTA curve (maximum at $320^{\circ} \mathrm{C}$ ). In the last phase, graphite oxidation occurs. The oxidation process starts at a temperature of 550-600 ${ }^{\circ} \mathrm{C}$ and lasts to end of the firing process. The exothermal nature of the graphite oxidation process is confirmed by a distinct maximum at the DTA curve. 


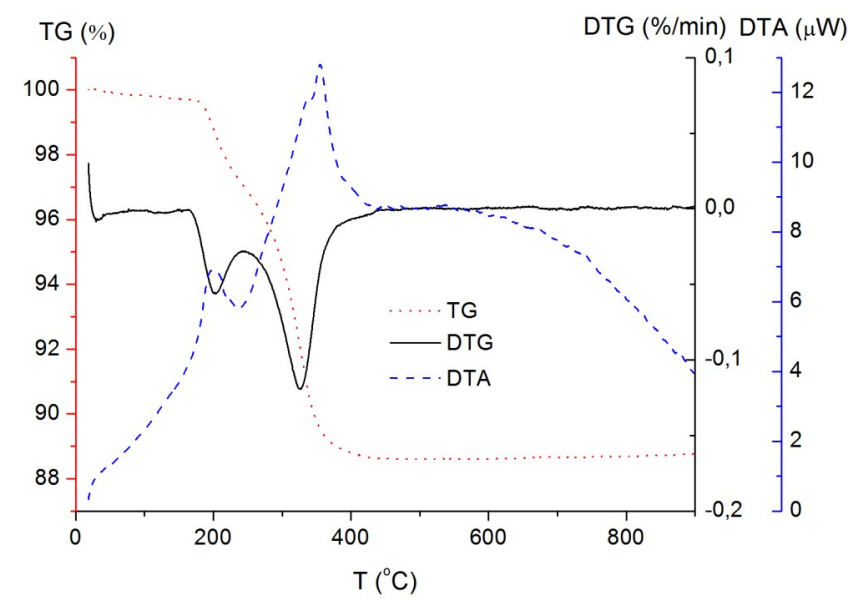

Figure 1. TG/DTG/DTA curves of the DP951 LTCC tape.

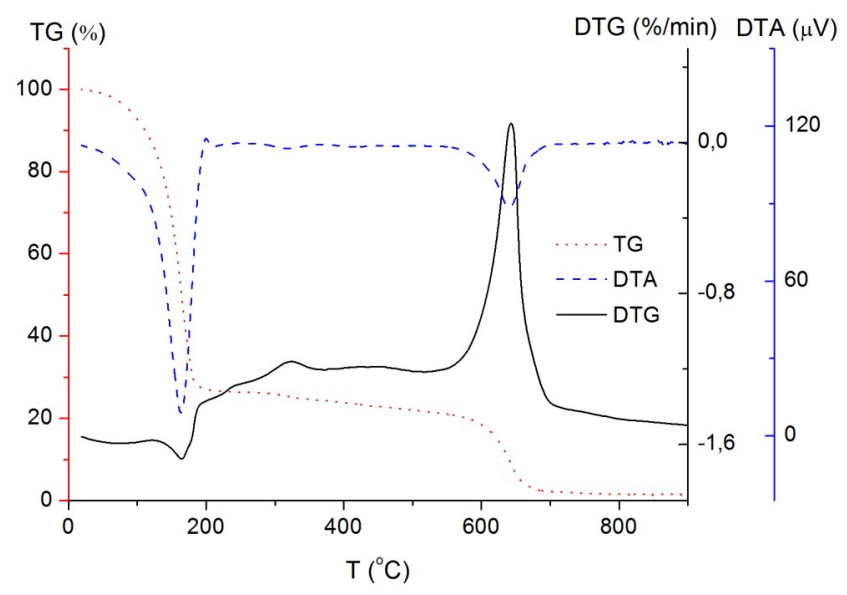

Figure 2. TG/DTG/DTA curves of the PVP-PG-G-based sacrificial paste.

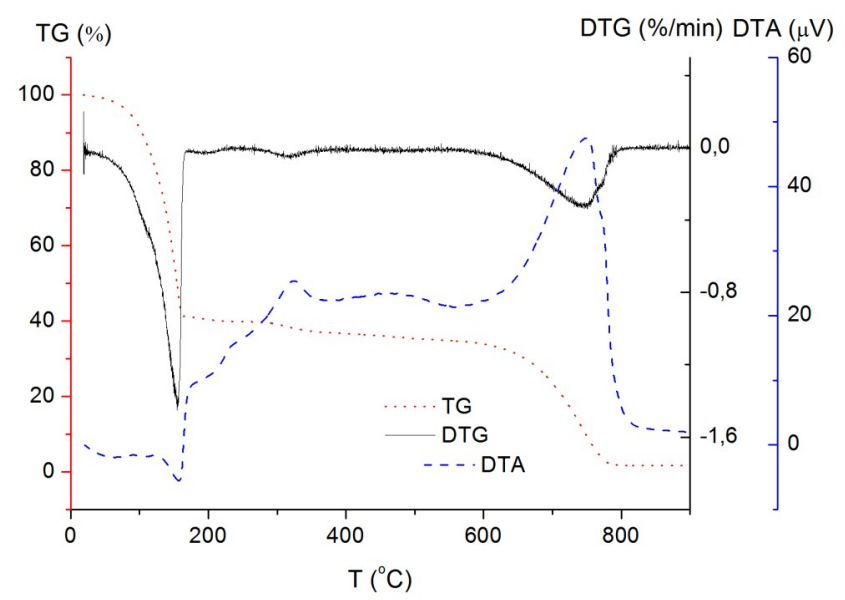

Figure 3. TG/DTG/DTA curves of the PVP-PG-TMP-based sacrificial paste. 


\subsection{Fabricated structures}

Both sacrificial pastes were applied to fabricate microchannels and $50 \mu \mathrm{m}$ thick membranes in LTCC substrate. The test structures were built of two DuPont (DP) 951 P2 and one DP 951 C2 LTCC tapes. Thickness of each unfired DP LTCC tape was equal to $165 \mu \mathrm{m}$ and 50 $\mu \mathrm{m}$, respectively. Fig. 4a-c presents each LTCC layer used to build the test structure. Two thicker layers (a) and (b) defines bottom of the test structure. The sacrificial carbon paste was deposited on layer (b). Deposited pattern consisted of five microchannels and three membranes. The microchannels had the same length $(15 \mathrm{~mm})$ and various widths $(0.3,0.5$, $0.75,1$ and $5 \mathrm{~mm}$ ). The diameters of the membranes were equal to $5,7.5$ and $10 \mathrm{~mm}$. In order to minimize the swelling of the membranes $[20,21]$ the additional escaping channels were added. Layer (c) covers deposited patterns and contains orifices for the escaping channels. The test structure was fabricated using well known "collate and laminate" technique [22]. Scheme of the applied "collate and laminate" technique is presented in Fig. 5. First two thicker LTCC tapes (DP 951 P2) tapes are laminated together using an isostatic press. The LTCC tapes were pressed with standard pressure of $20 \mathrm{MPa}$ and at temperature of $70^{\circ} \mathrm{C}$ for 10 min. Next the SVM paste was deposited on the LTCC laminate using screen-printing method through 200 mesh steel screen. After screen-printing, the SVM paste was dried for $10 \mathrm{~min}$ in air at $120^{\circ} \mathrm{C}$. Then, the LTCC laminate with deposited SVM was collated with $50 \mu \mathrm{m}$ thin LTCC layer by isostatic lamination. Parameters of the second lamination were similar to those applied during first one. Orifices for the escaping channels were cut in unfired DP 951 C2 tape by Nd-YAG laser (Aurel NAVS 30). In the last step, the test structures were co-fired in a box furnace (Nabertherm L3/S) with a standard two-step thermal profile. Plateau (dwell) for the debinding step was at $450^{\circ} \mathrm{C}$. The LTCC modules stayed in this stage for $1 \mathrm{~h}$ to ensure complete burnout of the organic constituents of the LTCC. After the debinding step, the temperature was increased up to $850^{\circ} \mathrm{C}$. The LTCC test structures were held at a peak temperature for 15 minutes. The applied graphite-based SVM pastes were removed during the co-firing process, leaving empty space in the LTCC test structures.

\subsection{X-ray photoelectron spectroscopy measurements}

XPS analysis was performed to investigate changes in atomic composition of the LTCC material after co-firing with PVP-PG-G and PVP-PG-TMP-based sacrificial pastes. Spectrometric analysis was performed for test structures with and without escaping channels and for reference LTCC structure. Before analysis the cover LTCC layer was removed from the PVA-PG-G and PVA-PG-TMP-based test structures. XPS scans were performed using SPES ESCA system equipped with a PHOIBOS 100 analyzer and SPECLAB software. The source of the X-rays was a magnesium anode $(10 \mathrm{kV}, 200 \mathrm{~W})$. The indicate angle was set to $90^{\circ}$ with respect to the test samples bottom surface. Wide scans were conducted with $30 \mathrm{eV}$, narrow scans with $5 \mathrm{eV}$ passage energy. The scanned area of $1.5 \mathrm{~mm} \times 1.5 \mathrm{~mm}$ was the same for all samples. The measured atomic compositions of the PVA-PG-G and PVA-PG-TMPbased test structures and the reference structure are given in Table 1. 

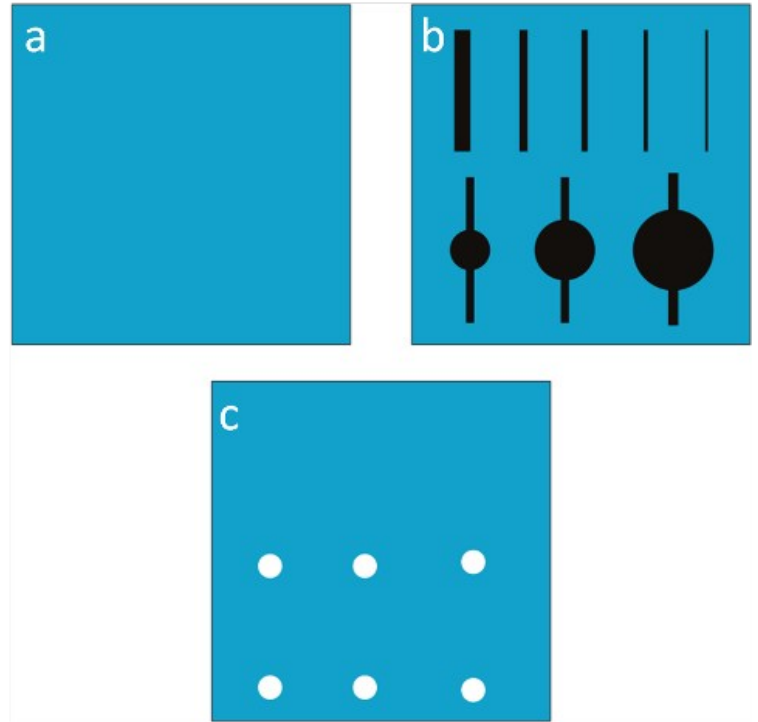

Figure 4. Layout of the final test structure.

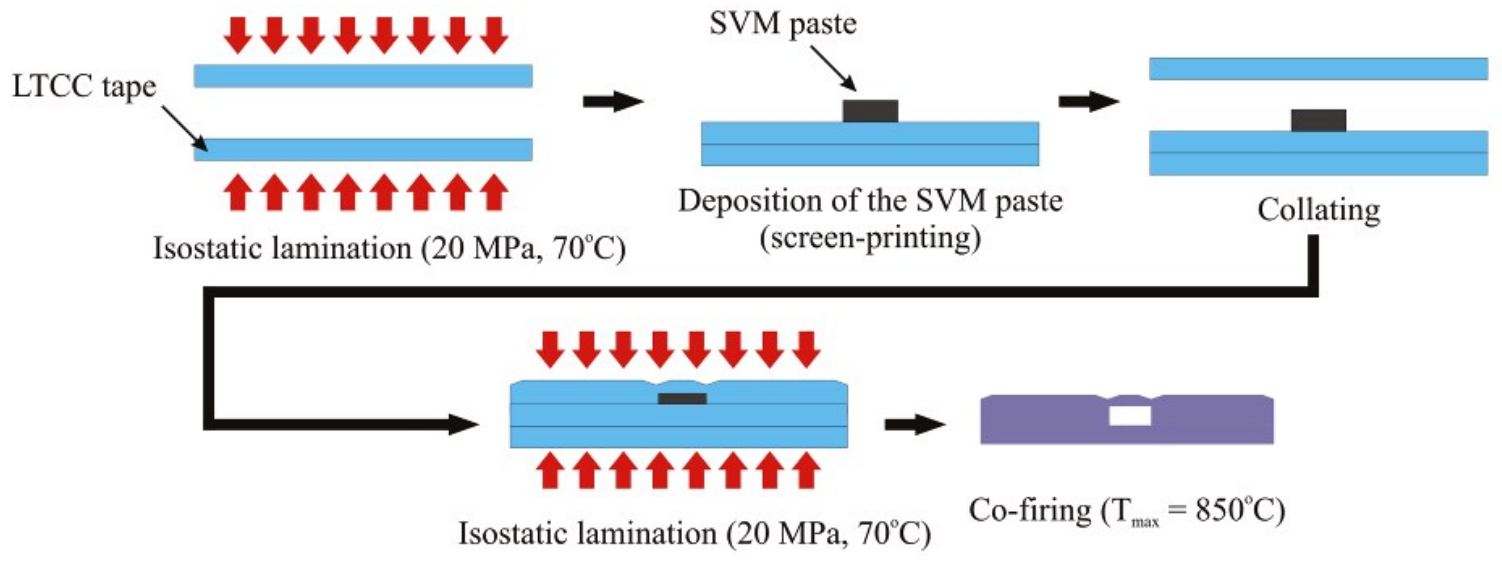

Figure 5. Flow chart of the "collate and laminate" process.

Table 1. Comparison of atomic composition of LTCC test structures surface.

\begin{tabular}{lccccc}
\hline Sample & C (\%) & O (\%) & Si (\%) & Al (\%) & Others (\%) \\
\hline $\begin{array}{l}\text { LTCC (reference) } \\
\text { PVP-PG-G }\end{array}$ & 2.8 & 61.3 & 14.0 & 12.4 & 9.5 \\
$\quad$ & & & & \\
$\quad$ With escaping channels & 3.4 & 64.5 & 13.1 & 10.6 & 7.4 \\
$\quad$ Without escaping channels & 17.4 & 54.8 & 13.5 & 11.0 & 3.3 \\
$\begin{array}{l}\text { PVP-PG-TMP } \\
\quad \text { With escaping channels }\end{array}$ & 4.3 & 68.0 & 11.5 & 10.4 & 5.8 \\
$\quad$ Without escaping channels & 17.6 & 55.6 & 12.6 & 10.8 & 5.4 \\
\hline
\end{tabular}


As can be seen, the atomic content of carbon was higher for a test structures made with PVAPG-G and PVA-PG-TMP carbon paste in respect to the reference LTCC structure. However, atomic content of carbon was evidently higher for test structures without escaping channels. The carbon content in the LTCC increased from about $2.8 \%$ to $3.4 \%$ and $4.3 \%$ for PVP-PG-G and PVP-PG-TMP-based structures, respectively. For test structures made without escaping channels the carbon content was much higher and was about $17.5 \%$.

\subsection{Surface wettability measurements}

The influence of the applied PVP-PG-G and PVP-PG-TMP sacrificial pastes on the LTCC surface wettability was analyzed using a goniometer (PG-X, Fibro System AB, Sweden). Static contact angle of distilled water were measured for bottom surface of the PVP-PG-G and PVP-PG-TMP-based test structures and reference LTCC structure. The contact angles were calculated as an average value of at least ten distilled water drops. Performed measurements shown that all investigated structures were characterized by similar wettability. Calculated contact angles were equal to $60^{\circ}, 62^{\circ}$ and $61^{\circ}$ for the reference LTCC, PVP-PG-G and PVP-PG-TMP-based structures, respectively. Similar wettability of the all studied structures confirms that both novel graphite pastes did not change the composition or morphology of the LTCC surface during technological process.

\section{Results and discussion}

\subsection{Preliminary studies on pastes}

Dissolution of both PVP grades into PG or BG proceeds easily, and none of the prepared vehicles exhibited a detectable tendency to gel, with some batches having been stored more than two years without showing any visible alteration. This is a considerable improvement over the previous PVA-PG-G- $\mathrm{H}_{2} \mathrm{O}$ vehicle $[19,20]$, which was more difficult to prepare and whose stability is too dependent on the $\mathrm{H}_{2} \mathrm{O}$ concentration (whose behavior in turn strongly depends on ambient humidity). On the other hand, while PG and BG both have low toxicity and similar (low) volatility, BG was found to be somewhat more aggressive, which was an issue on the thinnest $(50 \mu \mathrm{m})$ LTCC tapes. Therefore, PG was retained as the solvent for the rest of this work.

Next, the printability of a series of pastes using PVP-40 or PVP-360 was tested. As evidenced from Fig. 6, the low chain molecular weight (MW) of PVP-40 requires a high concentration to impart sufficient viscosity to the vehicle to avoid bleeding, which may expectedly also be achieved with much lower concentrations of PVP-360. However, both printable alternatives (high PVP-40 or low PVP-360) are not equivalent, as, for the same amount of graphite, there is a considerably higher amount (ca. $4 \times$ ) of binder to burn out during firing for PVP-40 than for PVP-360. Moreover, the volume of PVP-40 being roughly equal to that of the graphite powder, the dried layers are expected to be non-porous, which is of course not the case for PVP-360, where a small volume of binder merely holds the graphite particles together. 

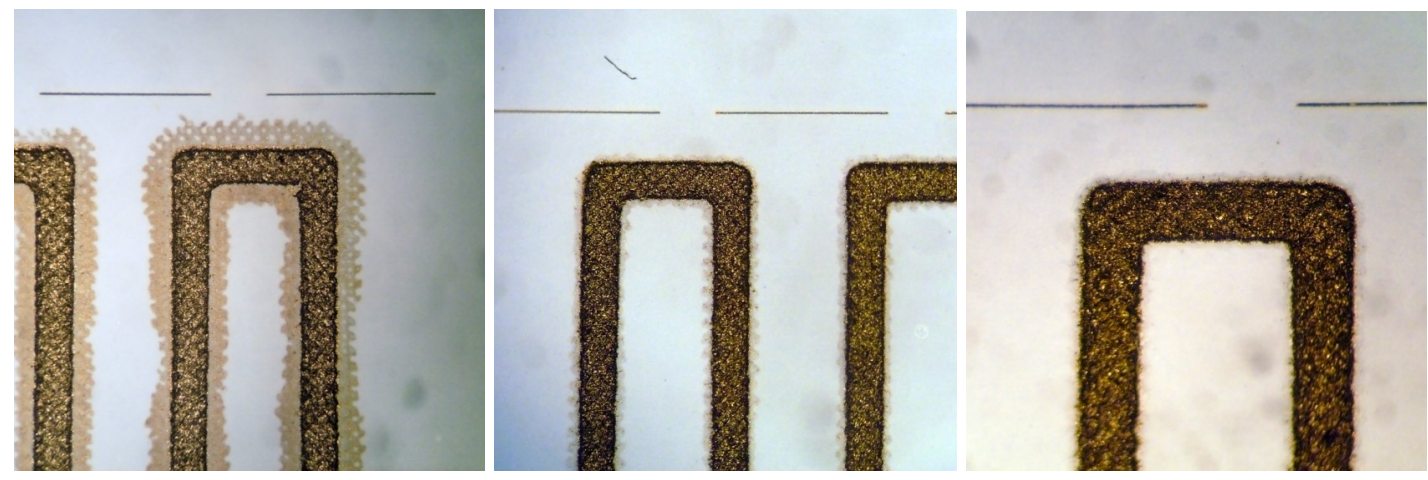

Figure 6. Printability tests, showing the positive effects on printability of increased PVP-40 concentration in vehicle. a) $17 \%$; b) $23 \%$; c) $26 \%$. Track width is $0.7 \mathrm{~mm}$.
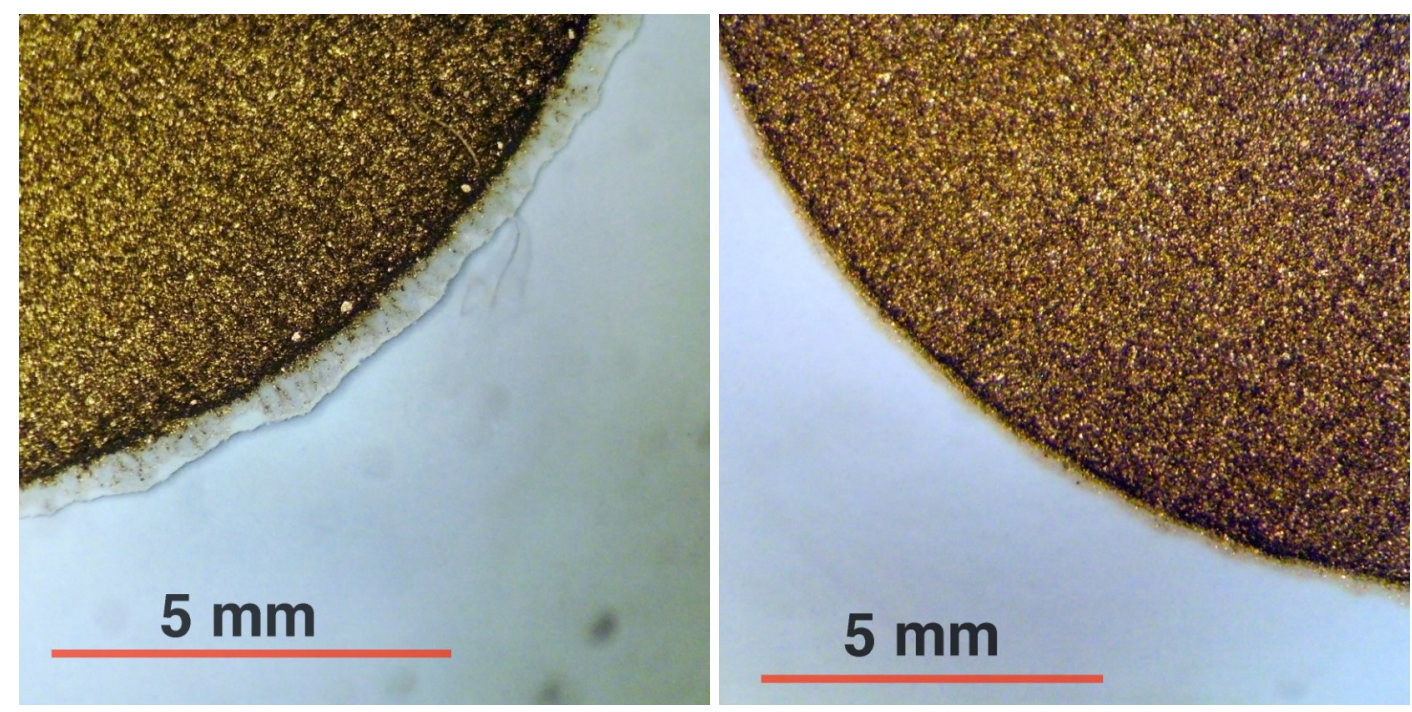

Figure 7. Effect of plasticizer on reliability when printing large-area SVM on thin $50 \mu \mathrm{m}$ DP 951 LTCC tape: a) without plasticizer, showing torn tape near membrane; b) with $\mathrm{G} \approx 1 / 4 \mathrm{PVP}$, showing intact tape.
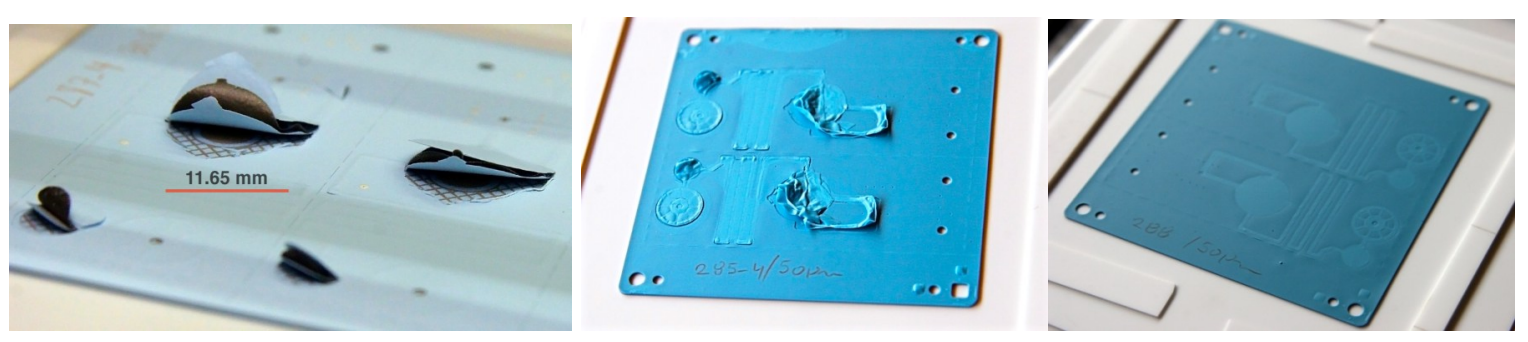

Figure 8. Effect of PVP:graphite volume ratio. a) 2:3, after debinding; b) 2:3, after firing; c) $1: 4$, after firing. 
The need for a plasticizer is illustrated in Fig. 7. Due to the high glass transition temperature of PVP $\left(T_{g} \approx 175^{\circ} \mathrm{C}\right)$ [23], thin LTCC tapes will be deformed after the drying stage by thermal stresses imparted by the hard SVM layer. In some cases, we even observed cracking at the edges of the large printed SVM areas that are needed for fabrication of membranes. Adding a small amount, from $1 / 4$ to $1 / 2$ of the binder depending on plasticizer, G, TMP or sorbitol efficiently addressed this issue. It must be noted that PVP always eventually becomes soft when stored in ambient atmosphere, due to its strong hygroscopicity and attendant plasticization by water; however this water goes away during the drying phase, hence the need for an additional plasticizer.

The last major encountered issue was linked with high PVP-40 resin concentrations. After seeing unusual degradation of first fired test structures, a special firing test was carried out to determine the critical step, interrupting the temperature rise at $520^{\circ} \mathrm{C}$, e.g. after debinding and before significant oxidation of graphite and sintering of LTCC. The results are shown in Fig. 8. Obviously, the large PVP-40 concentrations required for printability (binder : graphite volume ratio close to $2 / 3$, resulting in essentially airtight layers), coupled with the high degradation temperature of neat PVP [23], impede the outflow of decomposition products from the underlying LTCC tape, leading to the structures bursting open. Conversely, the higher chain length of PVP-360 allows well-printable inks with only ca. 1:4 PVP : graphite volume ratio, which results in porous dried SVM films and avoids this issue. Therefore, only inks based on PVP-360 were retained for further studies.

\subsection{X-ray and scanning electron microscope investigation of membranes and microchannels}

The PVA-PG-G and PVA-PG-TMP sacrificial pastes were used to fabricate membranes and microchannels in LTCC multilayer module. Membranes and microchannels are formed upon oxidation of the graphite-based pastes. The oxidation products (mainly $\mathrm{CO}$ and $\mathrm{CO}_{2}$ ) degas through porous LTCC material leaving empty space. According to performed X-ray and scanning electron microscope observations both applied graphite-based SVMs prevented deformations such as sagging or delamination of the fabricated membranes and microchannles. Applied SVM fillers ensured very good bonding between LTCC tape layers and mechanical support for spatial structures. Membranes and microchannels fabricated with two different sacrificial pastes were analyzed using X-ray tomography and scanning electron microscopy. Exemplary X-ray images of the membrane fabricated using PVA-PG-G and PVA-PG-TMP-based sacrificial paste are presented in Fig. 9. As can be seen from presented $\mathrm{X}$-ray images both fabricated membranes are continuous, flat and have very similar geometry. No delamination or deformations can be noticed. Similarly as for membranes, there was no significant difference between microchannels fabricated with both PVA-PG-G and PVA-PG-TMP-based sacrificial pastes. Exemplary SEM images of the microchannels fabricated with use of the SVM pastes are presented in Fig. 10a and b, respectively. As previously, applied SVM pastes avoided delamination between LTCC tape layers and retained geometry of the microchannels. Elliptical outline of the microchannel is consequence of the 
profile of the printed SVM paste and absence of the escaping orifices for paste oxidation products. It was noticed that microchannel height increases with its width for both investigated SVM pastes. Increased microchannel width results in higher swollen because of the higher amount of graphite oxidation products [21].

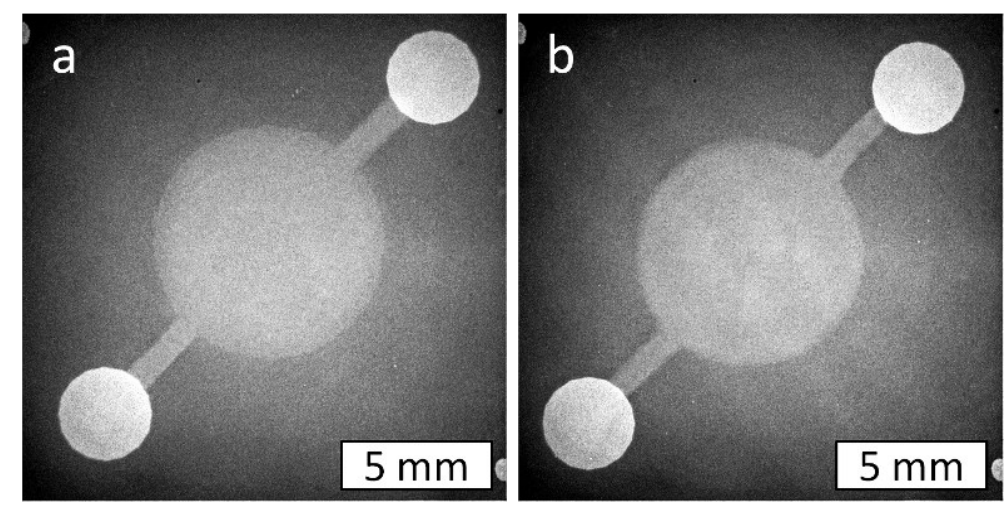

Figure 9. X-ray image 7.5 mm-diameter membrane made in LTCC using (a) PVP-PG-G and (b) PVP-PG-TMP-based sacrificial paste.

(a)

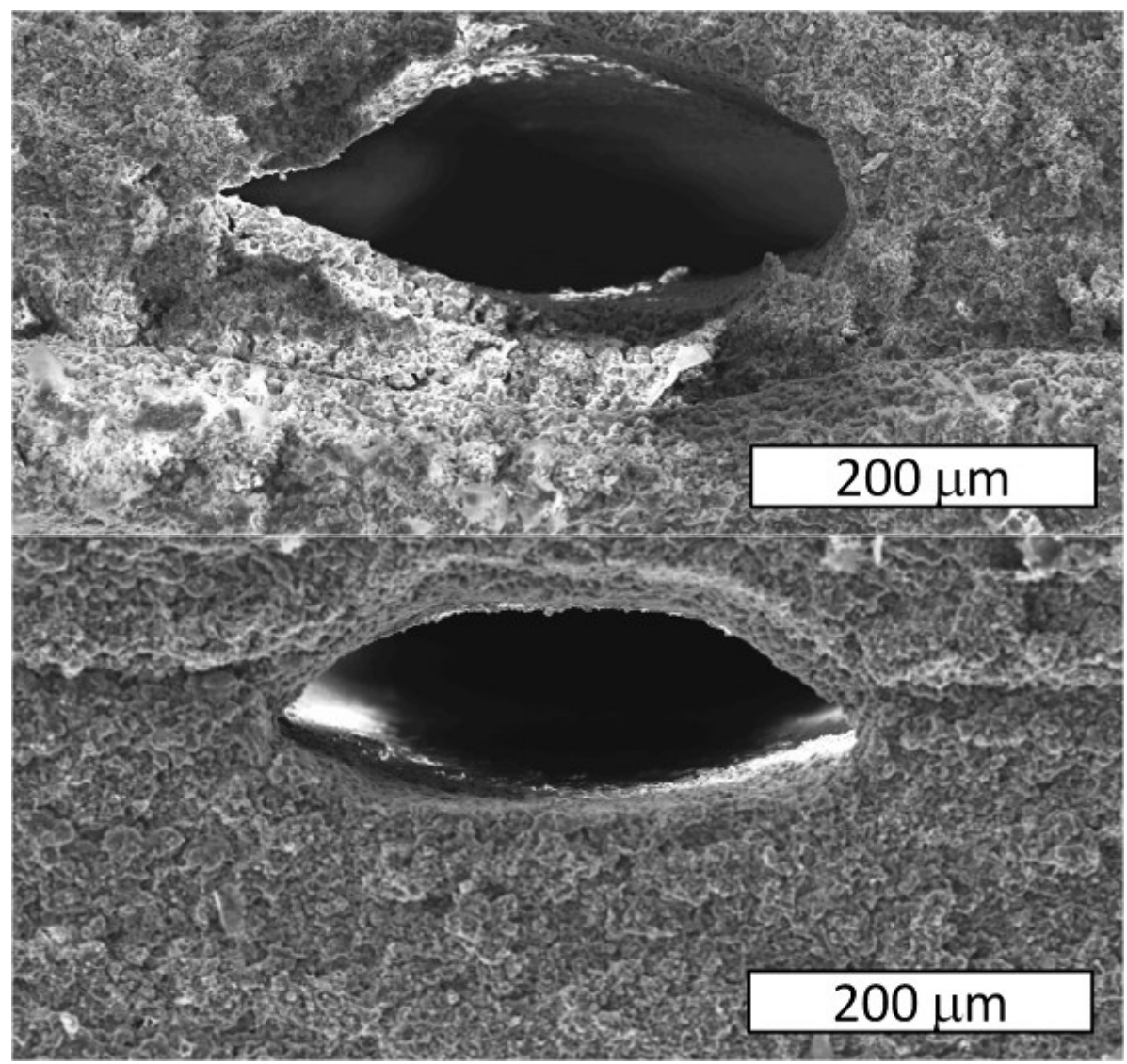

Figure 10. SEM images of the buried microchannels made in LTCC substrate using (a) PVPPG-G and (b) PVP-PG-TMP-based sacrificial paste. 


\subsection{Membrane profile measurements}

Profiles of the membranes fabricated with use of the PVA-PG-G and PVA-PG-TMP-based sacrificial pastes were measured using an optical profilometer (Veeco-Dektak 150 Surface Profiler). Measurements were done for membranes fabricated with and without escaping channels. Exemplary profiles of the membranes are presented in Figs. 11 and 12. As can be seen from Figs. 11a and 12a, lack of the escaping channels results in swollen membranes. According to the surface profile measurement the swelling increases with membrane diameter. Spacing for membranes without escaping channels fabricated with use of the PVA-PG-G and PVA-PG-TMP varies from $200 \mu \mathrm{m}$ to $375 \mu \mathrm{m}$ and from $180 \mu \mathrm{m}$ to $440 \mu \mathrm{m}$, respectively. Relationship between membrane spacing and its diameter for test structures without escaping channels is presented in Fig. 13. As distinct from membrane without escaping channels for membranes with escaping channels spacing remained more or less constant and was much smaller. It varied from ca. $7.5 \mu \mathrm{m}$ to about $8.5 \mu \mathrm{m}$ and was similar for membranes fabricated with use of PVA-PG-G and PVA-PG-TMP-based sacrificial paste. The relationship between membrane spacing and its diameter for test structures with escaping channels is presented in Fig. 14. Swelling and larger spacing for membranes without escaping channels can be explained by hindered oxidation of the graphite and poor degassing of the oxidation products through porous LTCC material. Pressure of the accumulated oxidation products swells partially sintered LTCC cover layer.

In order to investigate influence of the applied sacrificial paste type and presence/absence of the escaping channel on surface morphology of the fired LTCC, profile measurements were made for several samples: PVA-PG-G/TMP-based structures without escaping channels, PVA-PG-G/TMP-based structures with escaping channels and for the reference LTCC structure made with standard technological process. Before surface profile measurements the cover LTCC layer was removed from the test structures fabricated with use of the SVMs. As can be seen from Fig. 15 for test structures with escaping channels the SVM insert burnt away cleanly apart of applied graphite paste, however, for test structures without escaping channels contamination was noticed. According to the performed TGA analysis graphite oxidation depends on heating rate $[24,25]$, and can occur at temperature higher than LTCC densification temperature. Closing of the LTCC open-pores starts at $650{ }^{\circ} \mathrm{C}$ and ends at $900{ }^{\circ} \mathrm{C}$ [26], therefore, some part of graphite and its oxidation products can be trapped inside sintered LTCC structure, resulting in contamination [22]. 

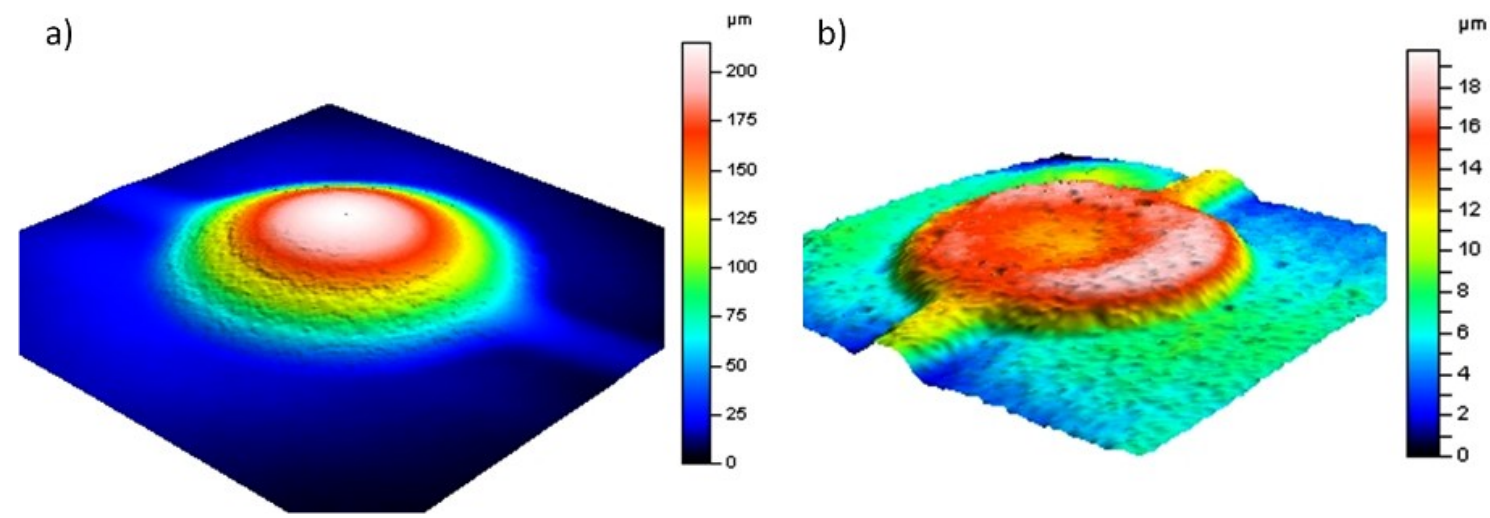

Figure 11. Surface profile of the membrane made in LTCC using PVP-PG-G-based sacrificial paste (a) without and (b) with escaping channel.
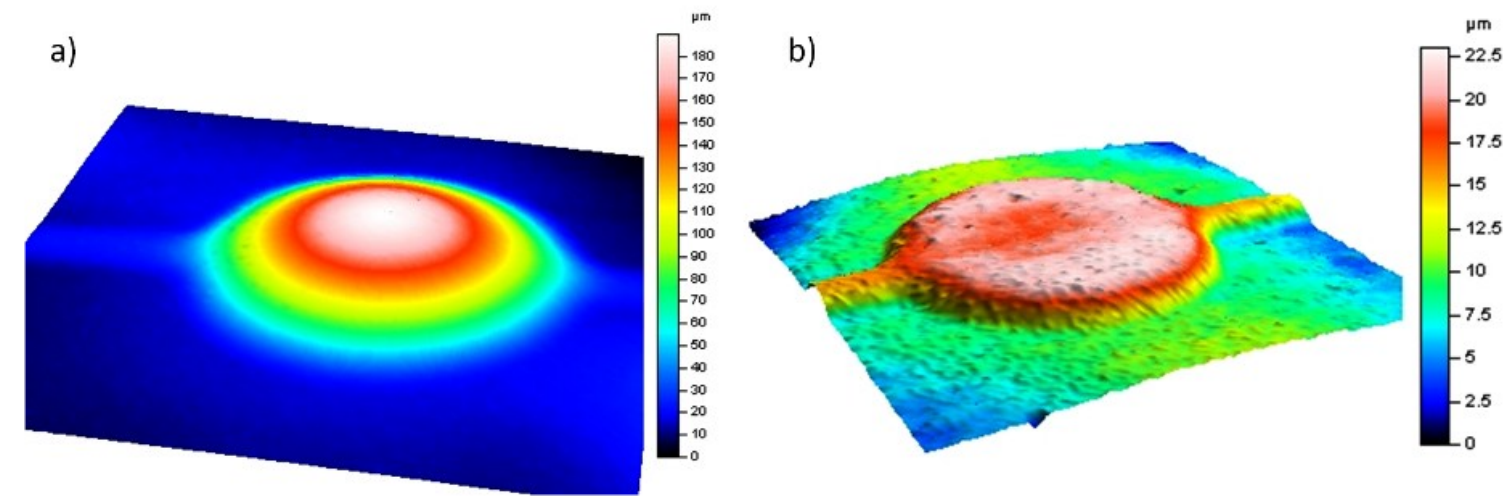

Figure 12. Surface profile of the membrane made in LTCC using PVP-PG-TMP-based sacrificial paste (a) without (b) with escaping channel.

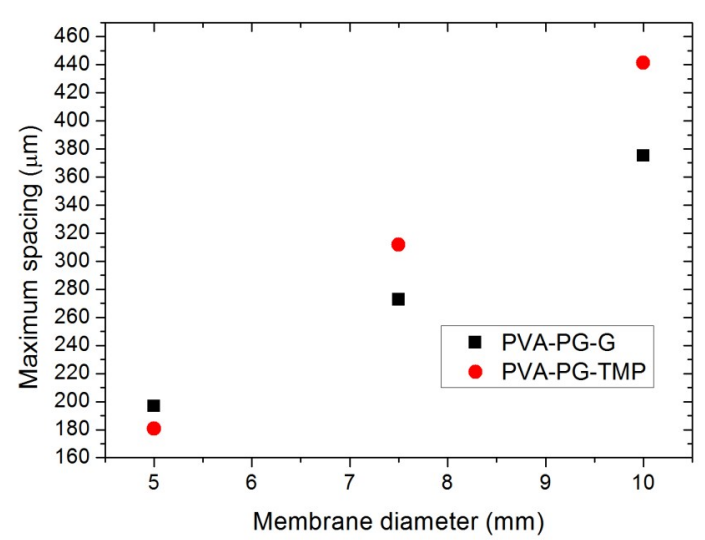

Figure 13. The dependence of membrane diameter on membrane spacing for test structure without escaping channels.

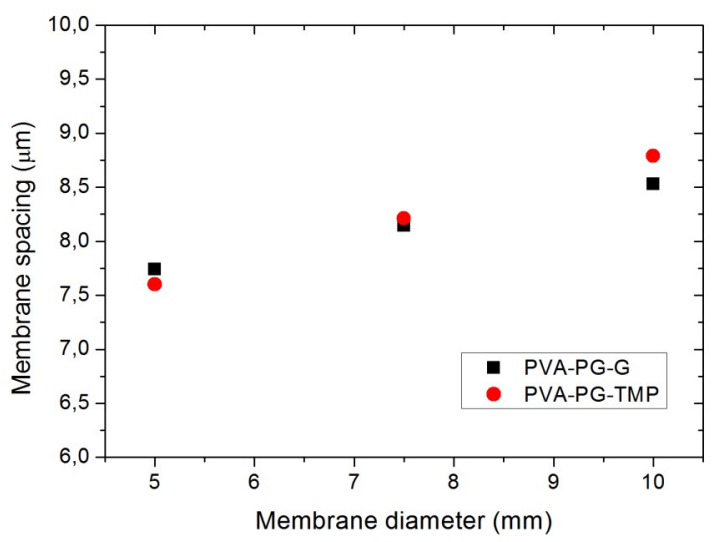

Figure 14. The dependence of membrane diameter on membrane spacing for test structure with escaping channels. 
(a)

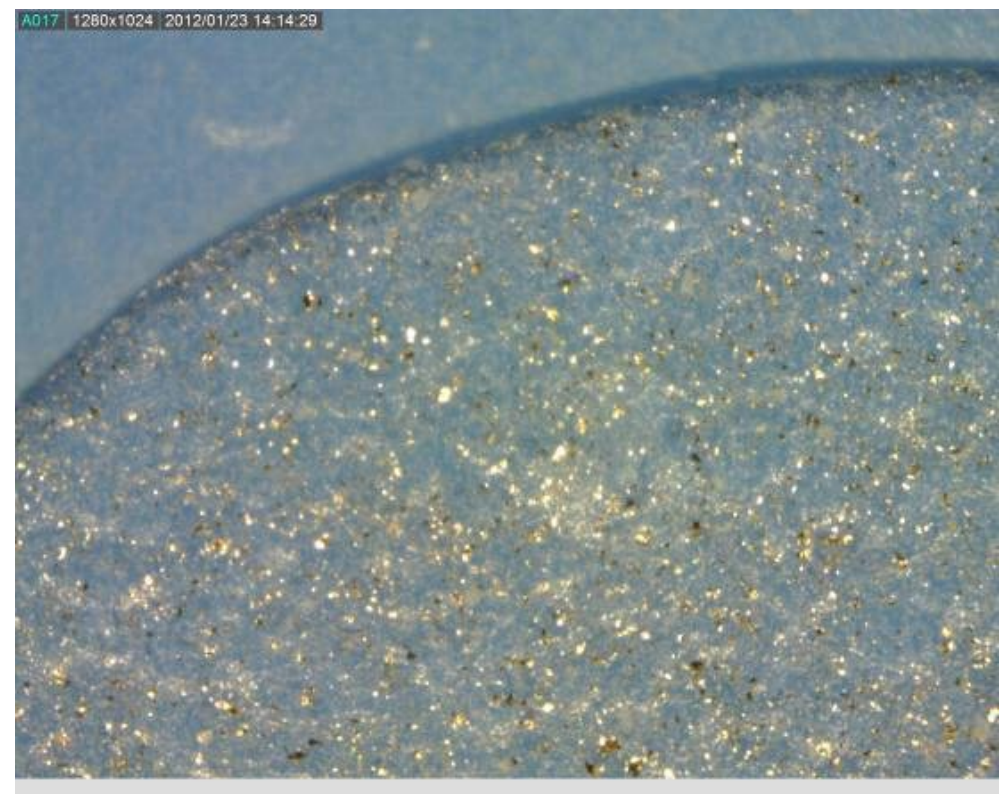

(b)

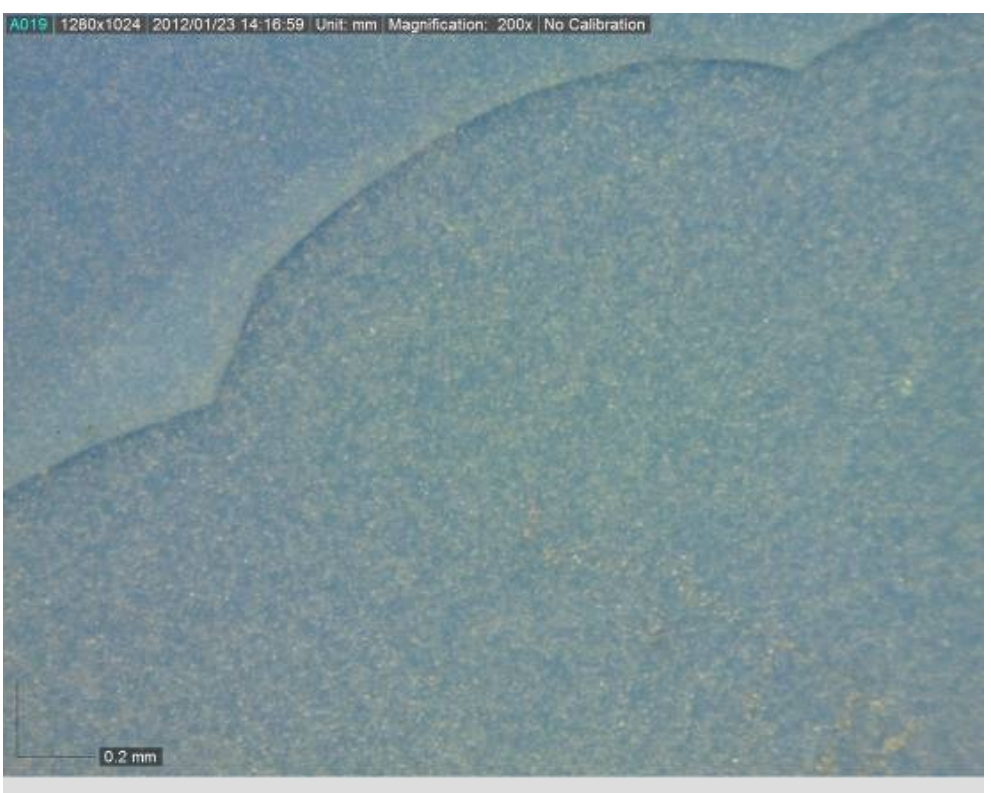

Figure 15. Surface of the LTCC test structure (a) without and (b) with escaping channels.

Table 2. Surface roughness of the LTCC test structures.

\begin{tabular}{llc}
\hline Sample & & $\mathbf{R}_{\mathbf{a}}(\boldsymbol{\mu} \mathbf{m})$ \\
\hline LTCC (reference) & & 0.231 \\
PVP-PG-G & with escaping channel & 0.242 \\
& without escaping channel & 0.304 \\
PVP-PG-TMP & with escaping channel & 0.223 \\
& without escaping channel & 0.281 \\
\hline
\end{tabular}


Surfaces of all investigated structures were scanned over $5 \mathrm{~mm}$ to measure the overall roughness using optical profilometer. Measured average surface roughness $\left(R_{a}\right)$ of the all investigated structures is given in Table 2. $R_{a}$ for the test structures with escaping channels made with use of the PVP-PG-G $(0.242 \mu \mathrm{m})$ and PVP-PG-TMP $(0.223 \mu \mathrm{m})$ was very similar to $R_{a}$ measured for the reference LTCC structure $(0.231 \mu \mathrm{m})$. Similar $R_{a}$ values obtained for the test structures with escaping channels and the reference structure confirm that both pastes do not damage the LTCC material during technological process. For the test structures without escaping channels, the corresponding measured $R_{a}$ was higher and was equal to $0.304 \mu \mathrm{m}$ and $0.281 \mu \mathrm{m}$ for PVP-PG-G and PVP-PG-TMP-based sacrificial paste, respectively. The higher surface roughness of the test structures without escaping channels confirms that some accumulated graphite residual products can be adsorbed on the bottom surface.

\section{Conclusions}

Two water-based sacrificial pastes were developed and applied for fabrication of thin membranes and microchannels in low temperature co-fired ceramics. The presented pastes were composed of graphite powder dispersed in an organic binder consisting of polyvinylpyrrolidone dissolved in a propylene glycol and plasticizer (glycol or trimethylopropane). Performed observations by scanning electron microscope and X-ray tomograph have shown that both presented sacrificial pastes are suitable for fabrication of thin membranes with diameters from $5 \mathrm{~mm}$ to $10 \mathrm{~mm}$ and buried microchannels with widths from $300 \mu \mathrm{m}$ to $5 \mathrm{~mm}$. However, observations made with use of x-ray tomography and scanning electron microscopy did not show significant differences between membranes and microchannels fabricated with PVA-PG-G and PVA-PG-TMP-based sacrificial pastes. Slight difference between membranes fabricated with PVA-PG-G and PVA-PG-TMP was noticed for profile measurements. Membranes fabricated with use of the PVA-PG-TMP were somewhat flat while PVP-PG-G-based membranes were bulged in the vicinity of their edge. This difference was noticed only for test structures with escaping channels. For test structures without escaping channels oxidation of graphite and degassing of the oxidation products were hindered and occurred only through porous LTCC material, which resulted in swollen membranes and caused contamination of the final structure. Contamination of the test structures without escaping channels was confirmed by optical observation and surface roughness measurements. For test structures with escaping channels both sacrificial pastes burned away cleanly during co-firing. Performed contact angle measurements and XPS analysis confirmed that both investigated graphite pastes did not change morphology or atomic composition of the LTCC material. Moreover, both presented sacrificial pastes are non-toxic and in case of a misprint they can be removed from the LTCC tape with water. 


\section{Acknowledgments}

The authors wish to thank the Polish National Science Centre (Grant No. N N515 607 639) and Wrocław University of Technology (Grant No. S10064 W12) for financial support, Mr. Matthias Garcin and Mr. Stanislas Wuilloud for help with sample fabrication, Dr. Yannick Fournier for photography and Dr. Piotr Markowski for optical profilometer measurments.

\section{References}

[1] Garron PE, Turlik I, Multichip module technology handbook. New York: McGraw-Hill; 1998.

[2] Unger M, Smetana W, Koch T, Radosavljevic G, High temperature characteristics of various LTCC-tapes. In: Proceedings, XXXIII International Conference of IMAPS Poland Chapter. 2009. p. 355-358.

[3] Kita J Dziedzic A Golonka L Bochenek A, Properties of laser cut LTCC heaters, Microelectronics Reliability 2000;40:1005-1010.

[4] Jacq C Maeder T Ryser P, Sensors and packages based on LTCC and thick-film technology for severe conditions, SĀDHANA - Academy Proceedings in Engineering Sciences 2009;34:677-687.

[5] Khanna PK, Hornbostel B, Burgard M, Schäfer W, Dorner J, Studies on threedimensional moulding, bonding and assembling of low-temperature-cofired ceramics for MEMS and MST applications, Materials Chemistry and Physics 2005;89:72-9.

[6] Fournier Y, Maeder T, Boutinard-Rouelle G, Barras A, Craquelin N, Ryser P, Integrated LTCC pressure/flow/temperature multisensory for compressed air diagnostics, Sensors 2010;10(12):11156-73.

[7] Sobociński M, Juuti J, Jantunen H, Golonka LJ, Piezoelectric unimorph valve assembled on an LTCC substrate, Sensors and Actuators A 2009;149:315-9.

[8] Ibáñez-Garcia N, Martinez-Cisneros CS, Valdés F, Alonso J, Green tape ceramics. New technological approach for integrating electronics and fluidics in microsystems, Trends in Analytical Chemistry 2008;27(1):24-33.

[9] Schindler K, Roosen A, Manufacture of 3D structures by cold low pressure lamination of ceramic green tapes, Journal of the European Ceramic Society 2009;29:899-904.

[10] Jurków D, Roguszczak H, Golonka LJ, Cold chemical lamination of ceramic green tapes, Journal of the European Ceramic Society 2009;29:703-9.

[11] Farhan Shafique M, Laister A, Clark M, Miles RE, Robertson ID, Fabrication of embedded microfluidic channels in low temperature co-fired ceramic technology using laser machining and progressive lamination, Journal of the European Ceramic Society 2011;31:2199-204.

[12] Birol H, Maeder T, Ryser P, Processing of graphite-based sacrificial layer for microfabrication of low temperature co-fired ceramics (LTCC), Sensors and Actuators A 2006;130-131:560-7.

[13] Malecha K, Golonka LJ, Microchannel fabrication process in LTCC ceramics, Microelectronics Reliability 2008;48:866-71. 
[14] Kinzy Jones W, Liu Y, Gao M, Micro heat pipes in low temperature cofire ceramic (LTCC) substrates, IEEE Transactions on components and packaging technologies 2003;26(1):110-5.

[15] Gongora-Rubio MR, Espinoza-Vallejos P, Sola-Laguna L, Santiago-Avilés JJ, Overview of low temperature co-fired ceramics tape technology for meso-system technology (MsST), Sensors and Actuators A 2001;89:222-41.

[16] Smetana W, Balluch B, Stangl G, Luftl S, Seidler S, Processing procedures for the realization of fine structured channels arrays and bridging elements by LTCCtechnology, Microelectronics Reliability 2009;49:592-9.

[17] Lucat C, Ginet P, Castille C, Debeda H, Menil F, Microsystem elements based on freestanding thick-films made with a new sacrificial layer process, Microelectronics Reliability 2008;48:872-5.

[16] Felten JJ, Compositions containing diethylene glycol ether, United States patent US4'070'200; 1978.

[19] Maeder T, Jacq C, Fournier Y, Ryser P, Formulation and processing of screen-printing vehicles for sacrificial layers on thick-film and LTCC substrates. In: Proceedings, XXXII International Conference of IMAPS Poland Chapter. 2008. p. B16.

[20] Malecha K, Maeder T, Jacq C, Ryser P, Structuration of the low temperature co-fired ceramics (LTCC) using novel sacrificial graphite paste with PVA-propylene glycolglycerol-water vehicle, Microelectronics Reliability 2011;51:805-11.

[21] Birol H, Maeder T, Ryser P, Application of graphite-based sacrificial layers for fabrication of LTCC (low temperature co-fired ceramic) membranes and microchannels, Journal of Micromechanics and Microengineering 2007;17:50-60.

[22] Peterson KA, Patel KD, Ho CK, Rohde SB, Nordquist CD, Walker CA, Wroblewski $\mathrm{BD}$, Okandan M, Novel microsystem applications with new techniques in lowtemperature co-fired ceramics, International Journal of Applied Ceramic Technology 2005;2(5):345-63.

[23] Kim SJ, Park SJ, Kim IY, Lee-YH, Kim-SI, Thermal characteristics of Poly(vinyl alcohol) and Poly(vinylpyrrolidone) IPNs, Journal of Applied Polymer Science 2002;86:1844-7.

[24] Birol H, Maeder T, Jacq C, Straessler S, Ryser P, Fabrication of low-temperature cofired ceramics micro-fluidic devices using sacrificial carbon layers, International Journal of Applied Ceramic Technology 2005;2(5):364-73.

[25] Khoong LE, Tan YM, Lam YC, Carbon burnout and densification of self-constrained LTCC for fabrication of embedded structures in a multi-layer platform, Journal of the European Ceramic Society 2009;29:457-63.

[26] Khoong LE, Tan YM, Lam YC, Study of deformation and porosity evolution of low temperature co-fired ceramic for embedded structures fabrication, Journal of the European Ceramic Society 2009;29:2737-45. 